\title{
Heat Transfer Analysis of Plate Fin Heat Sink with Dimples and Protrusions: Investigation of New Designs
}

\author{
Sharath Kumar S N ${ }^{1 *}$, Sathish $\mathrm{S}^{1}$, Purushothama $\mathrm{H} \mathrm{R}^{2}$ \\ ${ }^{1}$ Dr. Ambedkar Institute of Technology, BDA Outer Ring Road, Mallathalli, Bengaluru 560056, India \\ ${ }^{2}$ Siddaganga Institute of Technology, B H Road, Tumukuru 572103, India
}

Corresponding Author Email: sharathkumarsn.me@drait.edu.in

https://doi.org/10.18280/ijht.390621

Received: 24 October 2021

Accepted: 13 December 2021

\section{Keywords:}

heat sink, dimples, base temperature, thermal resistance

\begin{abstract}
Numerous works have been carried out in the design and analysis of Heat Sink, however hardly any work have been reported with studies on dimples and protrusions being added to the heat sink geometries. This paper aims to find the worthiness for addition of dimples and protrusions to the common plate fin heat sink geometries for heat transfer enhancement. Analysis was carried out in forced convention environment for 9 different velocities computationally for 12 heat sink geometries at constant heat flux boundary condition applied to base of heat sink. Decrease in base temperature and thermal resistance of heat sink was considered as the performance factors. Further these computational results were validated experimentally. The results obtained from computational analysis were in good agreement with experimental results. The research outcome show that addition of dimples has proved its worthiness at slightly higher velocity regime with thermal performance being increased for a dimpled heat sink as compared to non-dimpled heat sink. Therefore, currently developed dimpled heat sinks can be a potential model that can be used in forced convection environment for better thermal performance and economy.
\end{abstract}

\section{INTRODUCTION}

In the recent times, if analyzed, the major reason for failure of electronic device or system is due to overheating or thermal mismanagement. Whenever the electronic circuitry is powered on and electric charge flows through it, heat gets generated due to $I^{2} R$ losses or Joule heating (I- Current, R- Resistance). This heat has to be dissipated effectively so has to make these electronic devices reliable and operate in upright condition. In most of the cases dissipation of this heat naturally may not maintain the operating temperature of these electronic devices below the threshold value because of the processing capabilities that they have to deal with. This requires external attachments to boost up the heat transfer process. One such attachment is the use of heat sink. Heat sinks are the thermal devices having fins which are capable of absorbing and transferring heat. These heat sinks are attached to the electronic devices that require cooling by means of suitable thermal paste. The heat generated by these electronic devices is absorbed by the heat sink and is effectively transferred to the cooling medium, which is air in most of the cases. This heat carried away by the cooling medium can be further enhanced by supplying forced air from external source such as fan and blower. From past decade lot of design changes and researches, due to the intense interest among the researchers have witnessed the production of new and efficient heat sinks. In order to find an alternative and better solution for the removal of high heat flux from tiny area, in the year 1981, Tuckerman and Pease introduced the usage of heat sinks which has unique attributes such as high power dissipation to volume ratio, compactness and requirement of less quantity of coolant. From then oodles of researchers are working for the betterment of this design in terms of fin geometry, orientation and configuration. Some of the modern day designs include platefin, pin-fin, interrupting-fin, slotted-fin, inline-fin, staggeredfin, same and different height fin, same and different width fins, perforation-fins etc. For these designs to be effective, temperature distribution should be uniform, they should have low thermal resistance and the surface of the base should have lower temperature as possible. Apart from this as discussed earlier pumping power, compactness and less cost of fabrication are some of the essential requirements in considering the heat sink for a specific application. Other than the effect of shape of the fin for enhancement of heat transfer rate, there are other parameters that have been reported in the literature by many researchers. For instance, Elnaggar [1] has reported that the influence of thickness is more on the thermal performance as compared to fin numbers when the height of fins is kept constant. Wong and Indran [2] in their analysis showed that the fillet profile had a capability to improve the overall thermal performance of a plate fin heat sink with $U$ shaped channel profile being the best performer. It's also important to outline the effect of vortex generators as reported in Li et al. [3] where these extra attachments help in generation of vorticity, which increases the fluid mixing and in turn the heat transfer. Several other works have been reported where the generation of vortices has increased the heat transfer but with considerable amount of pressure drop. One such idea is the inclusion of dimples on the heat transfer surfaces. Bi et al. [4] analyzed the boundary layer disturbances taking place in the channel having dimples along with the pressure drop. Chang et al. [5] in their study showed that the increase in dimple height /depth increased the thermal performance of the dimple surfaces. Vorayos et al. [6] studied the effect of spacing 
between the dimples (pitch) and showed that the thermal performance parameter was increased by $26 \%$ for dimpled surfaces as compared to smooth surface. Gupta et al. [7] experimentally studied the thermal and flow performances of the heat sink with dimples. The effect of dimple pitch and dimple spacing at constant heat flux condition keeping dimple diameter same at the Reynolds number in the range of 6800 to 15200 was analysed. Results showed that there was significant increase in Nusselt number of dimple surface as compared to smooth surface. Further as reported by Won et al. [8] the presence of dimples helped in flow mixing which in turn increased the advection process. These studies were based on Nusselt number model and there are other models such as entropy generation optimization and pressure drop model which can be used for predicating the thermal performance.

Correlations based on numerical models have also helped in predicting the thermal performance of heat sinks to certain extent. Numerical correlations for total heat transfer and pressure loss co-efficient for a variable length channel was established by Biber [9]. Subsequent experimental analysis as carried on to validate Biber model with some changes and developments by Saini and Webb [10]. Other works include prediction of pressure drop and heat transfer co-efficient by the development of simple semi empirical model by Duan and Muzychka [11]. Apart from this detailed discussion on recent innovations in heat sink designs was reported by SN et al. [12].

However to the best of author's knowledge, the experimental and computational validation related to addition of dimples to heat sinks is not carried out. Also the comparative investigations of new designs with and without the addition of dimples has not been reported yet. This is of major importance because, the development of effective cooling solutions is critical in engineering applications, as excess heat can compromise the function of electronic systems. Some of the factors that has to be considered in dissipating heat effectively using heat sink are: Power to be dissipated, maximum allowable temperature, space or volume available for cooling, power density, pressure drop, bypass effects, air flow parameters, ease of fabrication and finally the cost. Increasing the velocity of air may increase the heat dissipation rate but at the expense of pressure drop and fan operating cost which includes the price of fan and energy required for it to run. Also, the addition of these fans which are operating at high speed can make the overall design of the electronic equipment bulky and may also increase unnecessary noise disturbances. This calls for the need of better design heat sink which can dissipate more heat keeping the operating cost as minimal as possible. One such design is the addition of concave and convex dimples on the plates of heat sink.

This work seeks to address the void specified in the previous paragraph by presenting experimental and numerical analysis comparing the thermal performance of flat plate heat sinks with and without dimple in flow through mode and constant heat flux boundary condition. The influence of this research work can be best appreciated by considering the limitations in the base temperature of heat sinks at high heat flux with respect to the pumping power involved. With the help of this study, it is feasible to enhance the thermal performance of plate fin heat sink. Since addition of dimples to current heat sink designs will have lower base temperatures as compared to non-dimpled heat sink for a given constant heat flux condition at a particular velocity. Further the addition of dimples to heat sinks will also reduce the pumping power required, as the pressure drop experienced by the dimpled heat sink will be lesser than the non-dimpled counterparts for the desired base temperature of the heat sink to be maintained. Therefore, the thermal performance of the Plate fin heat sinks can be effectively improved by choosing suitable analysis model with suitable design changes on sink side while making necessary changes on the coolant side such as change in flow direction, change in fluid property etc.

The scope of current analysis is to analyse the worthiness of addition of dimples to different design heat sink designs at constant heat flux boundary condition taking decrease in base temperature and thermal resistance of heat sink as the performance factor.

\section{METHODOLOGY}

Present work aims at understanding the worthiness of addition of dimples to flat plate heat sink. Computational approach is used for this purpose. Validation of this computational results was carried out by comparing it with experimental results.

\subsection{CFD analysis}

In the current investigation CFD model is developed to analyse heat transfer performance of different design plate-fin heat sinks with and without dimples. CFD analysis procedure involves three main stages. First stage is Pre-processing, which includes creation of desired model geometry and grid generation for the same. Later stage is solver execution, which involves feeding suitable boundary condition, making necessary solver setting and allowing the solver to perform the analysis. As expected, results are presented in the postprocessing stage.

In this paper total of 12 heat sink geometries were investigated. Figure 1 shows them as follows: 1- Flat Plate Heat Sink (FPHS), 2- Dimpled Flat Plate Heat Sink (DFPHS), 3- Step Flat Plate Heat Sink, 4- Dimpled Step Flat Plate Heat Sink, 5- Flared Flat Plate Heat Sink, 6- Dimpled Flared Flat Plate Heat Sink, 7- Fillet Flat Plate Heat Sink, 8- Dimpled Fillet Flat Plate Heat Sink, 9- Double Height Flat Plate Heat Sink, 10- Dimpled Double Height Flat Plate Heat Sink, 11Divergent Flat Plate Heat Sink, 12- Dimpled Divergent Flat Plate Heat Sink. The geometries were modelled using SpaceClaim 2020 R2 software. The base plate dimensions of the heat sink was kept same for all the 12 designs measuring $80 \mathrm{~mm} \times 80 \mathrm{~mm} \times 6 \mathrm{~mm}(1 \times \mathrm{w} \times \mathrm{t})$. While the maximum height of the plate fins was limited to $55 \mathrm{~mm}$ with the thickness measuring $0.7 \mathrm{~mm}$. Each heat sink design was modelled with and without dimples. The dimpled heat sinks were designed for dimple diameter to depth ratio of 0.5 with dimple diameter value of $4 \mathrm{~mm}$. Further the pitch between the dimples and spacing between the plates was maintained as shown in Figure 1. Test section's cross sectional dimensions was also varied to study the bypass effects, having dimensions of $57 \mathrm{~mm} \times 81$ $\mathrm{mm}(\mathrm{H} \times \mathrm{W}), 80 \mathrm{~mm} \times 81 \mathrm{~mm}$ and $80 \mathrm{~mm} \times 120 \mathrm{~mm}$. The properties considered for heat sink and air is given in Table 1.

ANSYS AUTODYN / Meshing software was used to generate the grid. Grid independency test was carried out for all the geometries to ensure accurate results at minimum computational time. Figure 2 shows the mesh domain of different heat sink chosen after carrying out grid independency test. Orthogonal quality was taken as the parameter to check the quality of the mesh. Care was taken such that the 
orthogonal quality is not below 0.1 , which is minimum threshold value for the grid / mesh to be confirmed as good quality. The constant heat flux of $18450 \mathrm{~W} / \mathrm{m}^{2}$ is applied to base of the heat sink. Variable inlet velocity i.e. $0.5,1.0,1.5$, $2.0,2.5,3.0,3.5,4.0,4.5 \mathrm{~m} / \mathrm{s}$ is applied at the inlet of the test section and pressure outlet boundary condition is applied to outlet of the test section. Analysis is carried out at 9 different velocities. Outer walls of the test section were treated as adiabatic walls so that no heat loss happens through the walls of the test section. Turbulence specification method was set to hydraulic diameter and turbulent intensity at both inlet and outlet. Hydraulic diameter and turbulent intensity were calculated as in ANSYS Fluent user guide.

Table 1. Air and Heat sink thermo-physical properties

\begin{tabular}{ccc}
\hline Properties & Air & Heat sink \\
\hline$\rho\left(\mathrm{kg} / \mathrm{m}^{3}\right)$ & 1.225 & 2705 \\
$C_{p}(\mathrm{~J} / \mathrm{kgK})$ & 1000 & 870 \\
$K(\mathrm{~W} / \mathrm{mK})$ & 0.0242 & 205 \\
$\mu(\mathrm{kg} / \mathrm{ms})$ & $1.789 \times 10^{-5}$ & - \\
\hline
\end{tabular}

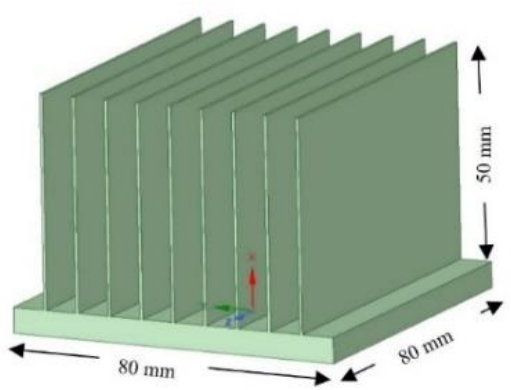

FPHS


DDHFPHS

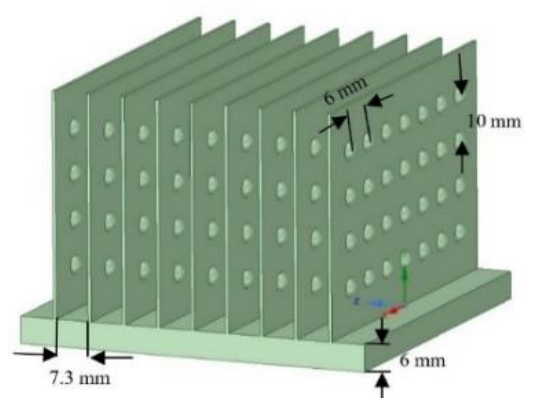

DFPHS



FFPHS
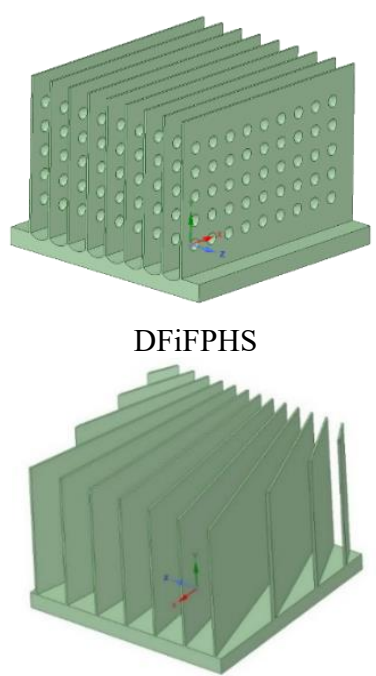

DiFPHS



SFPHS

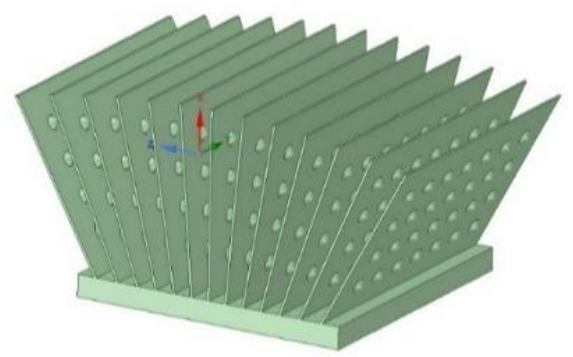

DFFPHS

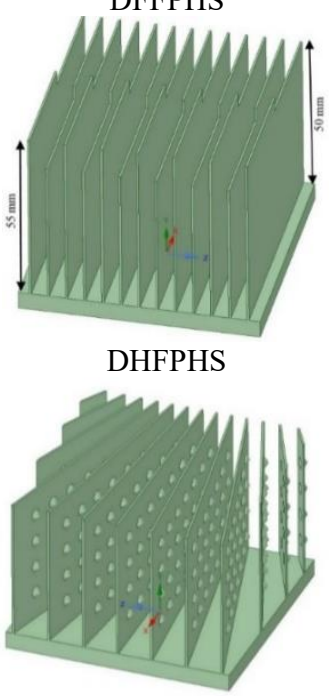

DDiFPHS

Figure 1. Schematics of different configuration heat sink

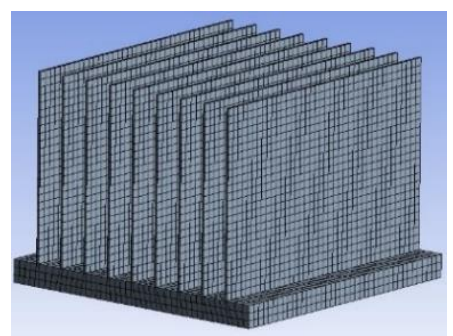

(a)

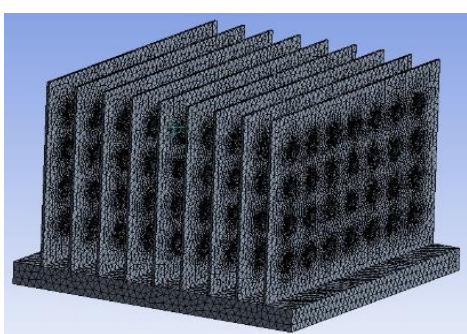

(b)

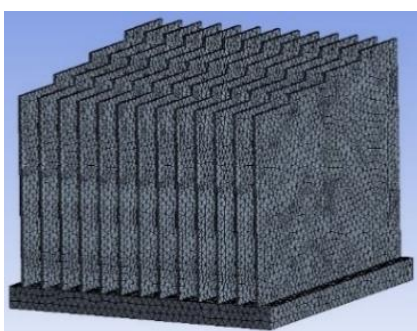

(c) 


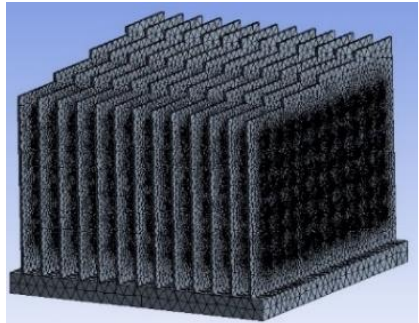

(d)



(g)

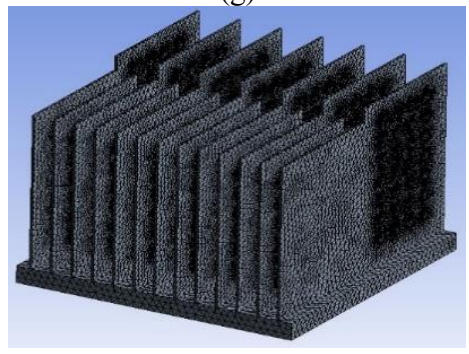

(j)

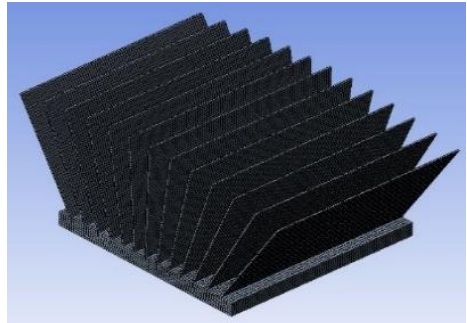

(e)



(h)

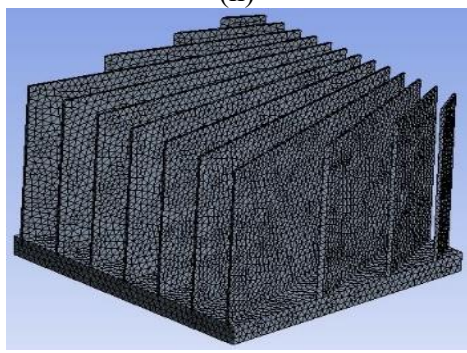

(k)

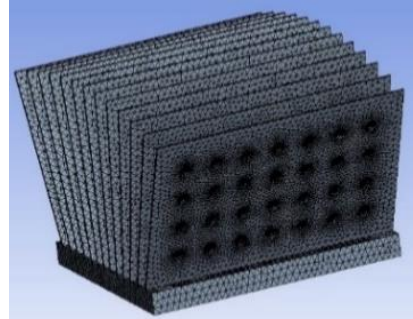

(f)

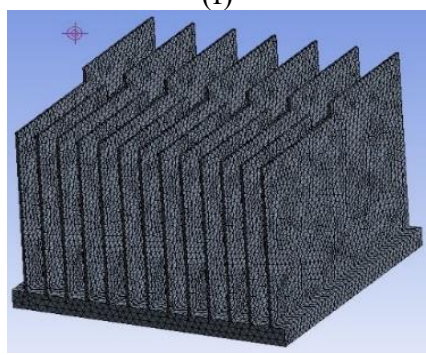

(i)

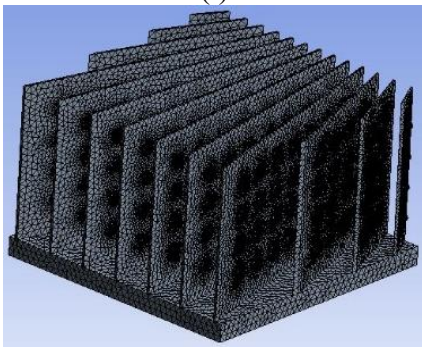

(1)

Figure 2. Mesh domain of different configuration heat sinks a) FPHS b) DFPHS c) SFPHS d) DSFPHS e) FFPHS f) DFFPHS g) FiFPHS h) DFiFPHS i) DHFPHS j) DDHFPHS, k) DiFPHS, l) DDiFPH

\subsection{Numerical modelling}

Continuity equation, Momentum equation i.e. the famous Navier-Stokes equation in short known as N-S equation and energy equation are numerically solved to simulate the heat transfer from the heat sinks considering some presumptions and simplifications as follows:

1. Properties of air depends on mean temperature.

2. Air flow is steady and incompressible.

3. Analysis is carried out as three dimensional conjugate heat transfer.

Continuity equation is given by:

$$
\nabla(\rho \vec{U})=0
$$

Momentum equation / $\mathrm{N}-\mathrm{S}$ equation across the three directions $x, y$ and $z$ are shown as follows:

$$
\begin{aligned}
& \nabla(\rho \vec{U} u)=-\frac{\partial p}{\partial x}+\frac{\partial \tau_{x x}}{\partial x}+\frac{\partial \tau_{y x}}{\partial y}+\frac{\partial \tau_{z x}}{\partial z} \\
& \nabla(\rho \vec{U} v)=-\frac{\partial p}{\partial y}+\frac{\partial \tau_{x y}}{\partial x}+\frac{\partial \tau_{y y}}{\partial y}+\frac{\partial \tau_{z y}}{\partial z} \\
& \nabla(\rho \vec{U} w)=-\frac{\partial p}{\partial x}+\frac{\partial \tau_{x z}}{\partial x}+\frac{\partial \tau_{y z}}{\partial y}+\frac{\partial \tau_{z z}}{\partial z}
\end{aligned}
$$

Energy equation is given by:

$$
\nabla(\rho h \vec{U})=-p \nabla \vec{U}+\nabla(\mathrm{k} \nabla \mathrm{T})+\varnothing+S_{h}
$$

\subsection{Solver setting}

3-Dimensional heat sink simulation was carried out at double precision using 8 parallel solver processes along with 1 solver GPGPUs per machine. HP Z800 workstation having hardware specification of Intel(R) Xeon (R) CPU (X5650 @ $2.67 \mathrm{GHz}, 12 \mathrm{CPUs}), 64 \mathrm{~GB}$ of RAM ( 8 x 8 GB DDR4) and 4 GB of Graphical memory (NVIDIA Quadro 5000) was used to carry out analysis. Pressure based coupled solver and second-order upwind interpolation is used to obtain steady state solution in ANSYS FLUENT 2020 R2. Pressure, momentum, turbulent kinetic energy and specific dissipation rate's pseudo transient explicit relaxation factors was set to $0.25,0.25,0.5$ and 0.5 respectively. For deeper convergence, continuity residual was set to 0.00001 . Hybrid initialization was performed which was followed by FMG initialization. FMG initialization provides a good approximate solution as a starting point for complex flow problems and also helps in model converging faster. Rest all the other parameters were set to default.

\subsection{Experimental approach}

Figure 3 shows the actual experimental setup and the parts are as follows: 1- Current Display, 2- Voltage Display, 3Temperature Display, 4-Voltage Regulator, 5- Heater Plate, 6Single Phase Centrifugal Blower, 7- Flow Control Valve, 8Digital Micro-meter, 9- Digital Anemometer, 10- Rectangular Duct. The setup consists of open circuit flow tunnel; comprising of single phase motorized blower. Ball type flow valve has been used to control the air flow into the duct. Duct having cross sectional dimension of $165 \mathrm{~mm} \times 100 \mathrm{~mm}$ and length $500 \mathrm{~mm}$ was used to test the heat sink. Heat sink was placed at a distance of $200 \mathrm{~mm}$ from the inlet, below which stainless steel heater pad having same dimension as that of the 
heat sink base plate was used. Thermocouples was used to measure the temperatures of heat sink walls and flowing fluid. Total of 10 thermocouples were used which include 2 at inlet, 2 at outlet, 2 on the heater pad and remaining 2 each on heat sink base plate and walls respectively. Heat loss from the walls of the duct was prevented by using mineral wool and asbestos sheets as the insulating material. Insulation to the duct walls becomes very much necessary to drive the heat flow through the heat sink from the heater pad which otherwise most of the heat would be conducted and convected through the duct walls. Digital Anemometer was placed at the entry of the test section to measure the flow velocity. Digital micro manometer was used to measure the differential pressure at inlet and outlet. Pressure probes were placed at entry and exit of the test section. Digital display for Temperature, Current and Voltage was provided to measure and control the operating conditions. Equipment specifications of the above mentioned equipments is mentioned in Table 2.

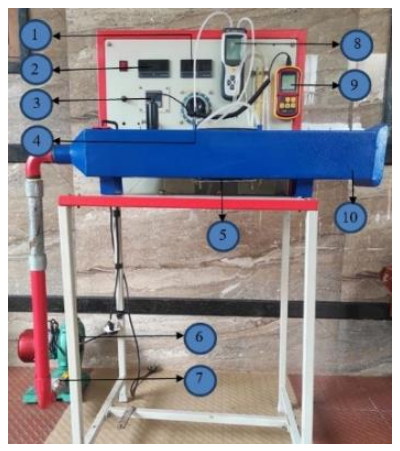

Figure 3. Experimental apparatus - actual photo

Table 2. Equipment specifications

\begin{tabular}{|c|c|}
\hline Instrument/Equipment & Specifications \\
\hline \multirow{8}{*}{ Blower } & Make: Speed \\
\hline & HP: 0.28 \\
\hline & Phase: Single \\
\hline & Volts: $220-240 \mathrm{~V}$ \\
\hline & Flow rate: $4800 \mathrm{cfm}$ \\
\hline & RPM: 2800 \\
\hline & Power Source: AC \\
\hline & Make: ADEPT \\
\hline Anemometer & Air velocity range: $0-30 \mathrm{~m} / \mathrm{s}$ \\
\hline & $\begin{array}{l}\text { Wind temp range: }-10^{\circ} \mathrm{C} \text { to } 45^{\circ} \mathrm{C} \\
\text { Make: Fluke }\end{array}$ \\
\hline Thermometer & Temperature range: $-30^{\circ} \mathrm{C}$ to $350^{\circ} \mathrm{C}$ \\
\hline (Contactless) & $\begin{array}{c}\text { Accuracy: } \pm 2.0 \% \text { of } \geq 0^{\circ} \mathrm{C} \\
\text { whichever is greater. }\end{array}$ \\
\hline k-type thermocouple (10 & Range: $-20^{\circ} \mathrm{C}$ to $400^{\circ} \mathrm{C}$ \\
\hline k-type thermocouple (10 & Sheath length: $20 \mathrm{~mm}$ \\
\hline & Sheath diameter: $1.2 \mathrm{~mm}$ \\
\hline Heater & $\begin{array}{c}80 \mathrm{~mm} \times 80 \mathrm{~mm}, 220 \mathrm{~V} \text { AC stainless } \\
\text { steel heating plate heater pad. } \\
\text { Power: } 2.5-4.5 \mathrm{~W} / \mathrm{cm}^{2} \\
\text { Thickness: } 3.5 \mathrm{~mm} .\end{array}$ \\
\hline
\end{tabular}

Experimental models (Figure 4, 5a and 5b) were fabricated using Aluminum 1050 material having density of $2.705 \times 10^{3}$ $\mathrm{kg} / \mathrm{m}^{3}$, Thermal conductivity of $205 \mathrm{~W} / \mathrm{m}^{\circ} \mathrm{K}$ and Co-efficient of thermal expansion of $2.36 \times 10^{-5} \mathrm{~K}^{-1}$. Creation of concave dimples on one side of the heat sink plate (Figure 5a) produced convex protrusions on the other side of the plate (Figure $5 \mathrm{~b}$ ) because of the thickness of the plate. So in the later stages of the work, for simplicity, instead of specifying the geometry as heat sink with dimples and protrusions, the name heat sink with dimples will be used.

Uncertainties in the measured parameters for the current experimental analysis is shown in Table 3. Regardless of the accuracy and precision all measurements have certain degree of uncertainty. It is observed that all the temperature readings measured fluctuated within $\pm 0.1^{\circ} \mathrm{C}$ after certain stabilized period. Also air velocity and pressure drop recorded fluctuations of $\pm 3 \%$ and $\pm 0.1 \mathrm{~Pa}$ respectively. These fluctuations can be due to physical equipment limitations.



Figure 4. FPHS (Experimental model)

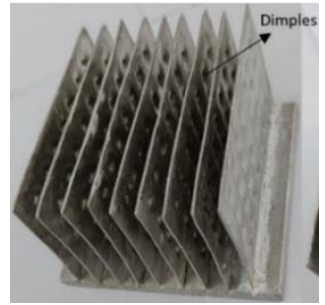

(a)

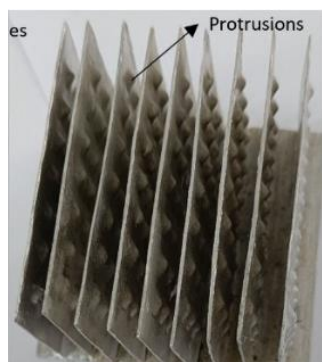

(b)

Figure 5. a. FPHS with dimples on one side of plate b. FPHS with protrusions on other side of plate

Table 3. Measurements uncertainty interval

\begin{tabular}{ccc}
\hline Measurements & Instruments used & $\begin{array}{c}\text { Uncertainty } \\
\text { Interval }\end{array}$ \\
\hline Temperature & Thermocouples & $\pm 0.1^{\circ} \mathrm{C}$ \\
Air velocity & Digital Anemometer & $\pm 3 \%$ \\
Pressure Drop & Digital Micro- & $\pm 0.1 \mathrm{~Pa}$ \\
& manometer & \\
Linear dimensions & Vernier caliper & $\pm 0.02 \mathrm{~mm}$ \\
\hline
\end{tabular}

\subsection{Calculation procedure}

In order to estimate the performance of the plate fin heat sink, thermal resistance and base temperature is considered.

Thermal resistance is given by

$$
R_{t h}=\frac{1}{\bar{h} A_{T}}
$$

where, $A_{T}$ is the total cooling area and $\bar{h}$ is the average heat transfer co-efficient.

Average heat transfer co-efficient is given by 


$$
\bar{h}=\frac{Q}{A_{T}\left(T_{b}-T_{m}\right)}
$$

where, $Q$ is the amount of heat transferred to the cooling medium i.e. air, $T_{b}$ is the base temperature of heat sink and $T_{m}$ is the mean temperature of air.

Mean temperature of air is given by

$$
T_{m}=\frac{\left(T_{a v g}+T_{b}\right)}{2}
$$

where, $T_{a v g}$ is the average temperature of air measures with respect to inlet and outlet temperature.

Average temperature of air is given by

$$
T_{\text {avg }}=\frac{\left(T_{\text {out }}+T_{\text {in }}\right)}{2}
$$

where, $T_{\text {out }}$ and $T_{\text {in }}$ are the temperatures measured at the outlet and inlet of the test section respectively.

Amount of heat transfer to the cooling medium is given by

$$
Q=m_{a} C_{p a}\left(T_{\text {out }}-T_{\text {in }}\right)
$$

where, $m_{a}$ and $C_{P a}$ refers to mass flow rate and the specific heat of air respectively.

It is also important to calculate the pressure drop across the heat sink in order to evaluate the additional pumping power required.

Pressure drop is given by

$$
\Delta P=P_{\text {in }}-P_{\text {out }}
$$

where, $P_{\text {in }}$ and $P_{\text {out }}$ refers to inlet and outlet pressure respectively.

\section{RESULT AND DISCUSSIONS}

Experiments and Numerical simulation were conducted to find the worthiness of addition of dimples to plate fin heat sink. Below sub sections brief out the results and understandings from the current analysis.

\subsection{Validation of computational results}

For fair comparison, boundary conditions, geometrical specifications, performance parameters and all the other operating parameters were considered exactly similar for both experimental and computational analysis. For the purpose of validation two geometrical models, FPHS and DFPHS was considered. Analysis was carried out at constant heat flux boundary condition of $18450 \mathrm{~W} / \mathrm{m}^{2}$ for 9 velocities in the range $0.5 \mathrm{~m} / \mathrm{s}$ to $4.5 \mathrm{~m} / \mathrm{s}$, with increments of $0.5 \mathrm{~m} / \mathrm{s}$.

Figure 6 and 7 shows the validation of CFD results for base temperature and thermal resistance respectively. It is evident from the Figures 6 and 7 that the experimental results and computational results are in good agreement. Both the curves in the above mentioned figures show similar trend and are almost identical. The values of thermal resistance and base temperature decreases with increase in velocity and their deviation between the computational and experimental results increases with increase in velocity. The maximum deviation is found to be $8.8 \%$ for base temperatures (Figure 6) and 9.64\% for thermal resistance at $4.5 \mathrm{~m} / \mathrm{s}$ (Figure 7) which are within the acceptable limits. The probable reason for the above noted difference is due to unavoidable thermal losses and measurement errors. Similarly the experimental and computational results were compared for heat sink without dimples and the maximum deviation for base temperature and thermal resistance was found to be within $9 \%$. Therefore, the developed computational approach is accurate enough and it can be effectively extended to study the worthiness of addition of dimples to various other heat sink geometries, maintaining all the other parameters same. In the current work, the results validated for above mentioned two geometries was extended to all the other heat sink geometries and is assumed to be correct since all the operating parameters considered remain same except geometry.



Figure 6. Validation of CFD results for Base temperature

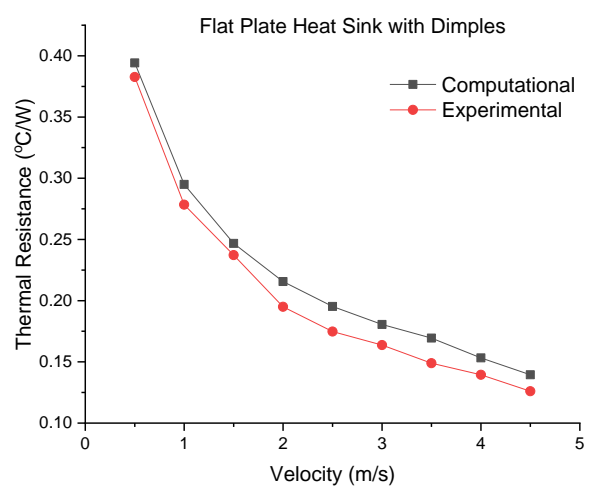

Figure 7. Validation of CFD results for thermal resistance

\subsection{Effect of duct dimensions / Bypass effect}

Table 4. Heat sink performance at different duct dimensions

\begin{tabular}{ccc}
\hline Case & $\begin{array}{c}\text { Duct C/S Dimension } \\
(\mathbf{m m} \text { x mm) }\end{array}$ & $\begin{array}{c}\text { FPHS Base Temperature } \\
(\mathbf{K})\end{array}$ \\
\hline 1 & $81 \times 170$ & 353.7231 \\
2 & $66 \times 100$ & 351.2442 \\
3 & $57 \times 82$ & 349.5926 \\
\hline
\end{tabular}

Current work also aims at understanding the effect of duct dimension, so 3 different $\mathrm{C} / \mathrm{S}$ ducts of dimension $81 \mathrm{~mm} \times 170$ $\mathrm{mm}, 66 \mathrm{~mm} \times 100 \mathrm{~mm}$ and $57 \mathrm{~mm}$ x $82 \mathrm{~mm}$ were designed. Analysis was carried out for simple FPHS geometry (Figure 1) at same velocity of $3.5 \mathrm{~m} / \mathrm{s}$ and constant heat flux of 18450 $\mathrm{W} / \mathrm{m}^{2}$ for all the 3 cases. As seen in the Table 4 , the performance of heat sink decreases with increase in duct crosssectional dimensions. This is due to the fact that air always takes least resistance path to flow and when the cross sectional 
area of the duct is more as in case 1, most of the air flows over the heat sink instead of flowing through the heat sink channels, there by carrying least amount of heat with it. On the other hand if the cross-sectional dimension is less, as in case of 3 , air particles are forced to pass through the heat sink channels, there by carrying most of the heat convected from plates of the heat sink, which results in increased heat transfer performance. Change in duct dimension from case 1 to case 3 has seen $24.57 \%$ decrease in thermal resistance and $7.68 \%$ decrease in base temperature of heat sink.

\subsection{Effect of heat sink geometries}

As stated earlier, in the current work, analysis is carried out for 12 designs, out of which 6 designs are common plate fin heat sinks and remaining 6 designs are the dimple versions of the above mentioned designs. Figure 8 shows the temperature contours of different configuration heat sinks at heat flux of $18450 \mathrm{~W} / \mathrm{m}^{2}$ and $4.5 \mathrm{~m} / \mathrm{s}$ velocity. It depicts that temperature at the upper sections is lower than the bottom section of heat sink for all the cases. The bottom section is in contact with the heat source and the top counterparts are in contact with cold air. Temperature boundary layer grows along the fin walls, with bottom part having more thermal boundary layer thickness. Disturbing the boundary layer intensifies the convection heat transfer. Addition of dimples serves this purpose. It is observed in Figure 8 that FPHS, SFPHS, FFPHS, FiFPHS, DHFPHS, DiFPHS has higher temperatures at bottom as compared to their dimpled counterparts respectively (DFPHS, DSFPHS, DFFPHS, DFiFPHS, DDHFPHS, DDiFPHS). DSFPHS, FFPHS and DDHFPHS has more uniform temperature distribution and lower temperatures at the bottom as compared with all the other cases. FPHS has the highest temperature at the bottom. But at very low velocities, addition of dimples shows higher temperature at the bottom as compared to its non-dimpled counterparts due to decrease in heat transfer caused by addition of dimples. It can be understood that while operating at low velocities, since the kinetic energy possessed by the fluid is less, the increment in disturbances in boundary layer, vorticity and mixing caused due to addition of dimples to heat sinks, couldn't compensate for the decrement in flow velocity and thereby the heat carrying capacity of fluid.

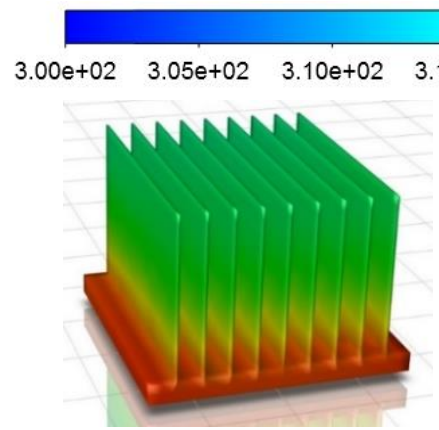

(m)

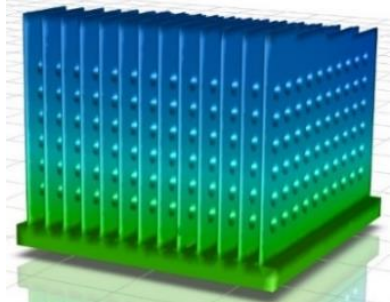

(p)

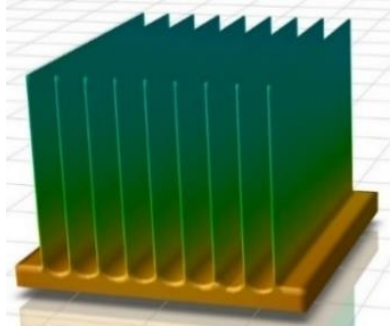

(s)



(v)

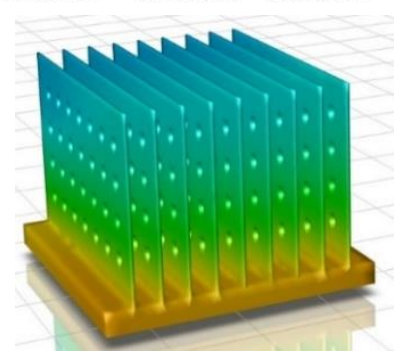

(n)

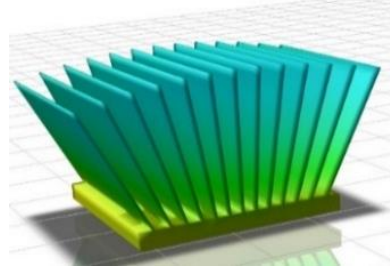

(q)



(t)

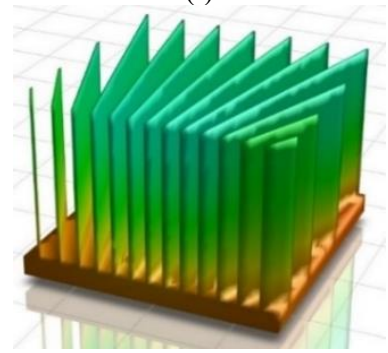

(w)

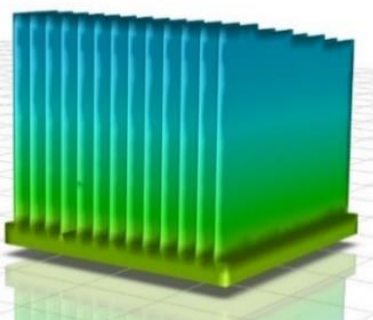

(o)

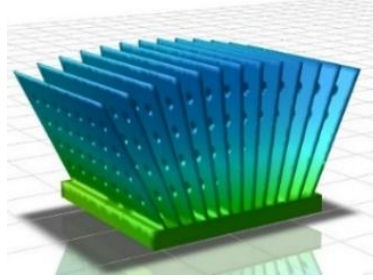

(r)

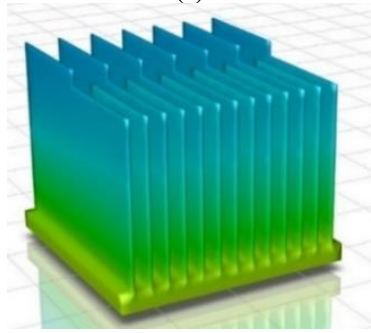

(u)

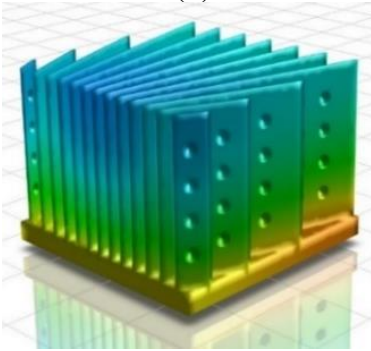

(x)

Figure 8. Temperature contours of different configuration heat sinks at $4.5 \mathrm{~m} / \mathrm{s}$ for $\mathrm{m}$ ) FPHS n) DFPHS o) SFPHS p) DSFPHS q) FFPHS r) DFFPHS s) FiFPHS t) DFiFPHS u) DHFPHS v) DDHFPHS, w) DiFPHS, x) DDiFPH 


\subsection{Effect on heat sink base temperature}

The most important criteria in the design of heat sink are to avoid overheating by reducing the temperature as much as possible. Figure 9 illustrates the variation of base temperature of heat sink as a function of velocity in case of all the dimpled and non-dimpled heat sink geometries considered earlier. The plots clearly show that base temperature of heat sink decreases with increase in velocity for all the geometries considered. All the plots obtained show the same trend and are identical. However, dimpled heat sinks possess relatively low base temperature as compared to non-dimpled heat sinks at slightly higher velocities. At velocities lesser than $1.5 \mathrm{~m} / \mathrm{s}$, nondimpled heat sinks exhibit slightly better thermal performance than dimpled heat sinks. For instance, comparing SFPHS and DSFPHS, at a velocity of $0.5 \mathrm{~m} / \mathrm{s}$, SFPHS has a base temperature value of $368.52 \mathrm{~K}$, while DSFPHS has a value of $375.77 \mathrm{~K}$. On the other hand, at a velocity of $4.5 \mathrm{~m} / \mathrm{s}$ SFPHS has base temperature value of $332.146 \mathrm{~K}$, while DSFPHS has a value of $327.22 \mathrm{~K}$.

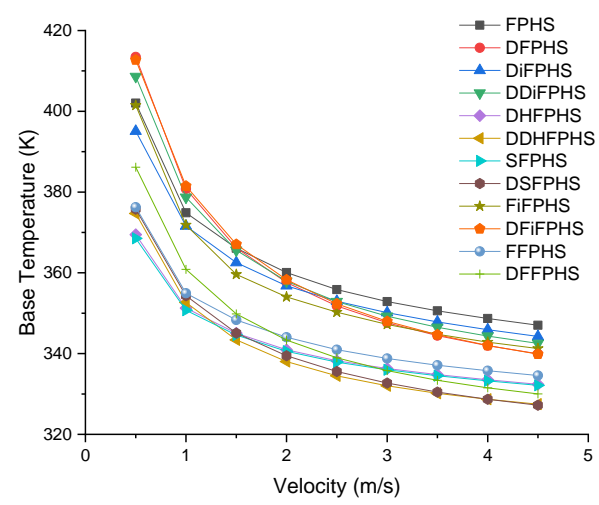

Figure 9. Variation of base temperature with velocity

\subsection{Effect on thermal resistance}

Figure 10 depicts the changes in thermal resistance versus velocity for various configurations of heat sinks considered. It indicates that thermal resistance reduces approximately exponentially with velocity for all the cases. Similar to base temperature, thermal resistance value for dimpled configurations is slightly higher at lower velocities and lower at higher velocities. The probable reason for the above performance variation at low and high velocity regime in addition to the one explained in the previous section is that, low velocity results in accumulation of air particles inside the dimple region. This is due to the fact that at low velocity regime energy possessed by the flowing fluid will be less and it would not have sufficient energy to overcome the surface bumps of dimples. This accumulation of air particles results in suppression of flow, thereby bringing down the heat carrying capacity of the air. On the other hand at high velocity regime fluid would have sufficient energy to overcome these surface bumps and the flow would be effortless without any suppression. Thereby carrying more amount of heat along with it. Among the 12 geometries analysed, it is also observed that DSFPHS has lowest base temperature and thermal resistance values of $327.22 \mathrm{~K}$ and $0.099^{\circ} \mathrm{C} / \mathrm{W}$ respectively, while highest base temperature belongs to FPHS and highest thermal resistance belongs to DiFPHS. DDHFPHS also show superior thermal performance having only $0.9 \%$ and $2 \%$ higher base temperature and thermal resistance values respectively as compared to DSFPHS. DFFPHS has $8.5 \%$ more base temperature and $13 \%$ lesser thermal resistance as compared with DSFPHS. It can be seen that FiFPHS has $12 \%$ lower base temperature and $32.22 \%$ lower thermal resistance as compared to FPHS, similar to the work reported by Hussain et al. [13]. Yet the addition of dimples to FiFPHS showed only marginal decrease in base temperature and thermal resistance

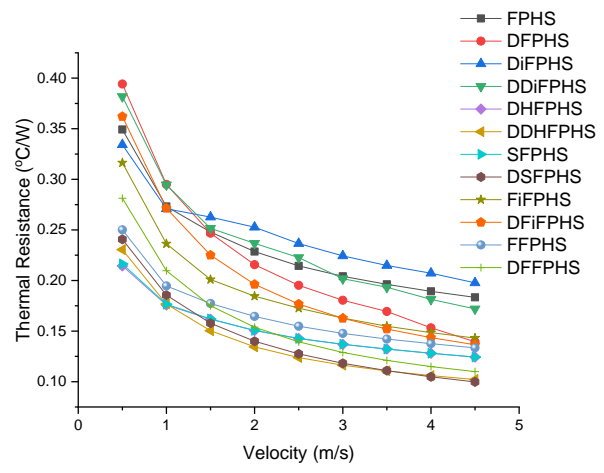

Figure 10. Variation of thermal resistance with velocity

\subsection{Effect on pressure drop}

Pressure drop across the heat sink geometry is one of the important factor to be considered in designing the heat sink, since it influences the operating cost and economics of heat sink directly. This is because, for higher pressure drops, cooling fluid has to be pumped at a higher pressure than required. Higher pumping power leads to higher operating cost. Figure 11 depicts the variation of pressure drop with respect to velocity for all the configurations of heat sink considered. As expected, the pressure drop value decreases with increase in velocity for all the cases. It is observed that DiFPHS and DDiFPHS configurations has the highest pressure drop values, while FFPHS and DFFPHS shows least pressure drop. This large difference in pressure for DiFPHS and DDiFPHS is due to the accumulation of air at the exit of heat sink. It is seen that DSFPHS, DDHFPHS, and DFiFPHS experiences $42.36 \%$, $31.62 \%$ and $6.8 \%$ more pressure drop respectively as compared to FFPHS.

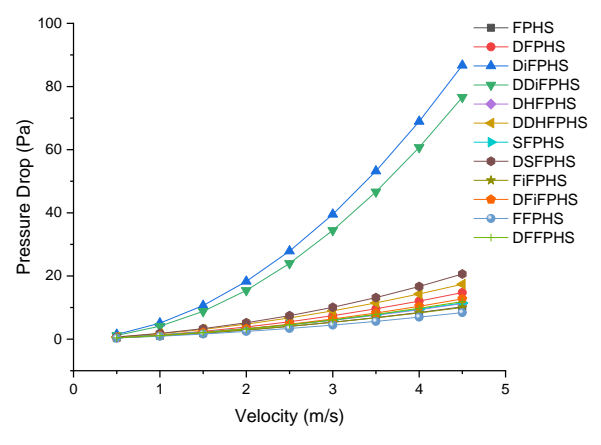

Figure 11. Variation of pressure drop with velocity

Even though the main reason for addition of dimples is to initiate more vorticity and mixing. This addition should not suppress the fluid flow and increase the pressure drop, thereby decreasing the local heat transfer and increasing the operational cost. For better heat transfer to happen the air has to keep flowing through the heat sink channels, taking away most of the heat from the plates with sufficient vorticity and 
mixing at minimal pressure drop. Further comparing the base temperatures of dimpled and non-dimpled geometry of stepped heat sink to check the worthiness of addition of dimples, in order to reduce the base temperature of heat sink to $332 \mathrm{~K}$, for dimpled geometry blower has to pump the air at the velocity of $3.0 \mathrm{~m} / \mathrm{s}$, but on the other hand, in order to reduce the base temperature of non-dimpled heat sink to same $332 \mathrm{~K}$ requires the blower to pump the air at a velocity of $4.5 \mathrm{~m} / \mathrm{s}$. Hence there is obvious saving in pumping power by the addition of dimples. Next, comparing the pressure drop values for dimpled and non-dimpled stepped heat sink geometries, in order to reduce the base temperature to $332 \mathrm{~K}$, the loss in pressure is found to be $13.875 \%$ more for non-dimpled heat sink compared to dimpled heat sink geometry. The thermal resistance value for a dimpled heat sink is found to be $24.683 \%$ less as compared to non-dimpled geometry.

In most of the cases heat sinks operate in forced convection environment as in case of computer processor cooling. The maximum operating temperature may reach up to $400 \mathrm{~K}$ but these processors need to be maintained at a temperature below $328 \mathrm{~K}$. Hence, addition of the dimple to heat sinks proves to be worthy as these dimpled heat sinks can effectively manage the temperature below the threshold limit utilizing minimal pumping power. The pumping power plays a very important role so as to keep the power consumption and the fan size as minimal as possible.

\section{CONCLUSIONS}

Experiments and Numerical simulation were conducted to find the worthiness of addition of dimples to plate fin heat sink. Analysis was carried out at 9 different velocities and at constant heat flux boundary condition for 12 geometries. The outcomes reveal that the experimental results and computational results are in good agreement. The maximum deviation between the two approaches is found to be $8.8 \%$ for base temperatures and $9.64 \%$ for thermal resistance under same conditions, which is within the acceptable limits. In particular, simulations were carried out to analyze the effect of duct dimensions, base temperature, thermal resistance and pressure drop on performance of flat plate heat sink due to addition of dimples. The study shows that the reduction in duct dimension has seen $24.57 \%$ decrease in thermal resistance and $7.68 \%$ decrease in base temperature of heat sink. Out of 12 geometries chosen for analysis, DSFPHS showed lowest base temperature while DFFPHS showed least pressure drop. DiFPHS with highest pressure drop and relatively higher base temperature and thermal resistance found to be the worst performer among all the cases considered. Further analysis carried out showed that addition of dimples had proved its worthiness only at high velocity regime $[>1.5 \mathrm{~m} / \mathrm{s}$ ] with thermal performance being increased up to $24.63 \%$ for a dimpled heat sink as compared to non-dimpled heat sink. At very low velocity regime [0.5 to $1.5 \mathrm{~m} / \mathrm{s}$ ] heat sinks without dimples proved to exhibit slightly better thermal performance than the dimpled heat sinks. Above results are contradictory to the basic heat transfer rule of increase in surface area increases the heat transfer. This brings us to the understanding that heat transfer is a phenomenon which depends on several other parameters and just by increase in surface area may not increase the heat transfer in all the cases. It should be considered that even a small decrease in temperature in electronic applications can be a favorable result in avoiding overheating of these devices. Further the practicality involved and the ease in fabrication of these dimpled heat sink also proves that they are worthy. Therefore, it can be concluded that dimples can be effectively used in heat sinks in forced convection environment in electronic cooling industry for better thermal performance and economical operation.

\section{REFERENCES}

[1] Elnaggar, M.H. (2015). Heat transfer enhancement by heat sink fin arrangement in electronic cooling. Journal of Multidisciplinary Engineering Science and Technology (JMEST), 2(3): 457-460.

[2] Wong, K.C., Indran, S. (2013). Impingement heat transfer of a plate fin heat sink with fillet profile. International Journal of Heat and Mass Transfer, 65: 1-9. https://doi.org/10.1016/j.ijheatmasstransfer.2013.05.059

[3] Li, H.Y., Chen, C.L., Chao, S.M., Liang, G.F. (2013). Enhancing heat transfer in a plate-fin heat sink using delta winglet vortex generators. International Journal of Heat and Mass Transfer, 67: 666-677. https://doi.org/10.1016/j.ijheatmasstransfer.2013.08.042

[4] Bi, C., Tang, G.H., Tao, W.Q. (2013). Heat transfer enhancement in mini-channel heat sinks with dimples and cylindrical grooves. Applied Thermal Engineering, 55(1-2):

121-132. https://doi.org/10.1016/j.applthermaleng.2013.03.007

[5] Chang, S.W., Chiou, S.F., Chang, S.F. (2007). Heat transfer of impinging jet array over concave-dimpled surface with applications to cooling of electronic chipsets. Experimental Thermal and Fluid Science, 31(7): 625-640 https://doi.org/10.1016/j.expthermflusci.2006.06.008

[6] Vorayos, N., Katkhaw, N., Kiatsiriroat, T., Nuntaphan, A. (2016). Heat transfer behavior of flat plate having spherical dimpled surfaces. Case Studies in Thermal Engineering, 8 :

370-377. https://doi.org/10.1016/j.csite.2016.09.004

[7] Gupta, A., Kumar, M., Patil, A.K. (2019). Enhanced heat transfer in plate fin heat sink with dimples and protrusions. Heat and Mass Transfer, 55(8): 2247-2260. https://doi.org/10.1007/s00231-019-02561-w

[8] Won, S.Y., Mahmood, G.I., Ligrani, P.M. (2003). Flow structure and local Nusselt number variations in a channel with angled crossed-rib turbulators. International Journal of Heat and Mass Transfer, 46(17): 3153-3166. https://doi.org/10.1016/S00179310(03)00103-0

[9] Biber, C.R. (1997). Pressure drop and heat transfer in an isothermal channel with impinging flow. IEEE Transactions on Components, Packaging, and Manufacturing Technology: Part A, 20(4): 458-462. https://doi.org/10.1109/95.650935

[10] Saini, M., Webb, R.L. (2002). Validation of models for air cooled plane fin heat sinks used in computer cooling. In ITherm 2002. Eighth Intersociety Conference on Thermal and Thermomechanical Phenomena in Electronic Systems (Cat. No. 02CH37258), 243-250. https://doi.org/10.1109/ITHERM.2002.1012464

[11] Duan, Z., Muzychka, Y.S. (2007). Pressure drop of impingement air cooled plate fin heat sinks. Trans. ASME, $\quad 129(2)$ : https://doi.org/10.1115/1.2721094

190-194.

12] SN, S.K., S, S., Purushothama, H.R. (2019). Recent 
developments in the design of Heat Sinks for heat transfer enhancement-A review. International Journal of Science, Technology, Engineering and Management-A VTU Publication, 1(4): 1-8.

[13] Hussain, A.A., Freegah, B., Khalaf, B.S., Towsyfyan, H. (2019). Numerical investigation of heat transfer enhancement in plate-fin heat sinks: Effect of flow direction and fillet profile. Case Studies in Thermal Engineering, 13 :

100388. https://doi.org/10.1016/j.csite.2018.100388

\section{NOMENCLATURE}

$\vec{U}$

Velocity of fluid, $\mathrm{ms}^{-1}$ p Pressure, $\mathrm{Pa}$

$u, v, w$ Velocity component in $x, \mathrm{y}, \mathrm{z}-$ direction

respectively, $\mathrm{ms}^{-1}$

$h \quad$ Aggregate enthalpy

$K \quad$ Thermal conductivity, $\mathrm{Wm}^{-1} \mathrm{~K}^{-1}$

T Temperature, $\mathrm{K}$

$S_{h} \quad$ Source term

\section{Greek symbols}

$\phi \quad$ Dissipation term

$\mu \quad$ dynamic viscosity, $\mathrm{kg} \cdot \mathrm{m}^{-1} \cdot \mathrm{s}^{-1}$

$\rho \quad$ density $\left(\mathrm{kg} / \mathrm{m}^{3}\right)$

$\tau \quad$ Viscous stress tensor term 